\title{
Personality profiles in adults with attention deficit hyperactivity disorder (ADHD)
}

\author{
Nader Perroud ${ }^{1,2^{*}}$, Roland Hasler ${ }^{1}$, Nicolas Golay ${ }^{1}$, Julien Zimmermann ${ }^{1}$, Paco Prada', Rosetta Nicastro ${ }^{1}$, \\ Jean-Michel Aubry ${ }^{1,2}$, Stefano Ardu ${ }^{3}$, François R Herrmann ${ }^{4}$, Panteleimon Giannakopoulos ${ }^{1,2}$ and Patrick Baud ${ }^{1}$
}

\begin{abstract}
Background: Previous studies suggested that the presence of ADHD in children and young adolescents may affect the development of personality. Whether or not the persistence of ADHD in adult life is associated with distinct personality patterns is still matter for debate. To address this issue, we compared the profiles of the Temperament and Character Inventory (TCI) that assesses personality dimensions in 119 adults ADHD and 403 controls.

Methods: ANCOVA were used to examine group differences (controls vs. ADHD and ADHD inattentive type vs. ADHD combined + hyperactive/impulsive types) in Temperaments and Characters. Partial correlation coefficients were used to assess correlation between $\mathrm{TCl}$ and expression and severity of symptoms of ADHD.

Results: High novelty seeking (NS), harm avoidance (HA) and self-transcendence (ST) scores as well as low self-directedness (SD) and cooperativeness (C) scores were associated with ADHD diagnosis. Low SD was the strongest personality trait associated with adult ADHD. Cases with the ADHD inattentive type showed higher HA and lower SD scores compared to the combined and hyperactive/impulsive types. High HA scores correlated with inattention symptoms whereas high NS and ST scores were related to hyperactive symptoms. Finally low SD and high NS were associated with increased ADHD severity.
\end{abstract}

Conclusions: Distinct temperaments were associated with inattentive versus hyperactive/impulsive symptoms supporting the heterogeneous nature of the disorder.

Keywords: ADHD, TCl, Attention, Hyperactivity, Impulsiveness, Personality

\section{Background}

Attention-deficit hyperactivity disorder (ADHD) is characterized by impaired attention control, impulsiveness and hyperactivity (motor restlessness). This disorder affects 3 to $7 \%$ of children and persists in adulthood in roughly 30 to $60 \%$ of subjects [1]. A deleterious familial environment in childhood as well as perinatal adverse events have been frequently reported in adult ADHD cases and are thought to have a negative impact on personality features [2]. Earlier studies in this field revealed that adults with ADHD tend to be more pessimistic, introverted, rebellious, and aggressive [3], show high levels of psychological distress, dissatisfaction, low capacity to

\footnotetext{
* Correspondence: nader.perroud@hcuge.ch

'Department of Mental Health and Psychiatry, University Hospitals of Geneva, Geneva, Switzerland

${ }^{2}$ Department of Psychiatry, University of Geneva, Geneva, Switzerland

Full list of author information is available at the end of the article
}

be organized and self-disciplined, and are less sociable, altruistic and sympathetic to others [2, 4].

Among the tools used to assess personality, the Temperament and Character Inventory (TCI), a self-report questionnaire assessing four dimensions of temperament (novelty seeking (NS), harm avoidance (HA), reward dependence (RD) and persistence $(\mathrm{P})$ ) and three dimensions of character (self-directedness (SD), cooperativeness (C) and self-transcendence (ST)), based on the Cloninger's biopsychosocial theory of personality, has aroused growing interest in ADHD subjects [5]. Its development was based on the assumption that personality construct can be described as a combination of a small number of temperament and character dimensions. Reflecting basic emotional responses to external and/or internal stimuli, temperaments are moderately heritable, and fairly stable throughout life $[6,7]$. They have been hypothesized to 
play a role in the development of psychopathology in several psychiatric disorders including ADHD $[8,9]$.

Characters are believed to represent more complex cognitive processes and refer to individual differences in goals, self-concepts and values. Being tightly linked to environmental influences, character dimensions may vary as a function of developmental stages during lifespan.

Previous studies on temperaments in adults and children suffering from ADHD led to highly comparable data showing high scores on NS, conceived as the tendency to be impulsive and irritable versus rigid and stoical, high scores on HA, defined as the tendency to be pessimistic and anxious versus optimistic and risk-taking, low scores on $\mathrm{P}$, assessing the tendency to be persevering and ambitious versus easily discouraged and indolent, and low scores on RD defined as the tendency to be sociable and warm versus aloof and cold [4, 10-21].

In terms of character patterns, earlier data reported that ADHD subjects differ from controls by low SD, referring to the tendency to be blaming and inept, low $\mathrm{C}$ associated with hostility and opportunism, and high ST describing people who are intuitive and insightful [10-12, 15-18, 21, 22]. Most of these temperament and character changes in ADHD subjects support the idea of low psychological functioning [16].

However, ADHD is not a clinically homogeneous condition. In recent years, three main subtypes (according to DSM-V) have been recognized, the predominantly inattentive presentation if the subject mainly has symptoms of inattention, the predominantly hyperactive-impulsive presentation if mainly symptoms of hyperactivityimpulsivity are present, and the combined presentation where both criteria of inattention and hyperactivityimpulsivity are present. To date, several studies have investigated which personality dimension is associated with specific subtypes of the disorder however leading to controversial data $[10,13,15,17,20,23-26]$. Overall these studies linked deviation in NS to hyperactive/ impulsive symptoms and deviation in HA to inattentive symptoms although deviation in almost all of the temperaments and characters have been reported.

Some studies using the Five Factor Model (FFM) of Personality also investigated the link with the severity of ADHD, but this has been poorly explored using the TCI leading to conflicting data [27]. For instance, Salgado et al. [13] found that the combined subtype of ADHD was associated with higher scores in NS and lower scores in $\mathrm{C}$ and HA than the predominantly inattentive subtype. They also found an inverse relationship between the number of inattentive symptoms and SD scores and a positive correlation with HA scores, whereas the number of hyperactive/ impulsive symptoms were positively correlated with NS and P scores. Lynn et al. [15] found that NS and the character dimension $\mathrm{C}$ accounted for the majority of variability in symptoms of hyperactivity-impulsivity while inattention was mainly predicted by NS. Faraone et al. [17] found that hyperactive/impulsive and inattention symptoms positively correlated with NS and HA scores and negatively with RD, P, SD and C scores. Gomez et al. [23], in a population based study of ADHD, found association between inattention and HA and between hyperactive/impulsive symptoms and P. Wood et al. [24] observed in children a genetic correlation between NS and hyperactive/impulsive symptoms. In adolescents, Young et al. [25] showed that attention and hyperactive/impulsive symptoms were mainly explained by NS. Van Dijk et al. [20] in a study comparing subjects suffering from borderline personality disorder to ADHD subjects, found that high NS was associated with the inattention symptoms of ADHD. In a twin study, Merwood et al. [26] found that NS was genetically associated with both symptoms of inattention and hyperactivity/impulsivity and that HA was genetically associated with inattention only. They also found some evidence of genetic association between $\mathrm{P}$ and the two ADHD domains (inattention and hyperactivity/impulsivity) suggesting that different profiles of temperament are genetically linked to ADHD dimensions in adults. Purper-Ouakil et al. [10] also found evidence showing that temperaments and characters were related to specific symptoms of ADHD. In their nonclinical sample of children, attention problems were negatively correlated with SD whereas NS was related to externalized symptoms and less to attentional problems. $\mathrm{C}$ was also inversely correlated with externalized symptoms. In the clinical sample (ADHD subjects) NS was linked to impulsivity/hyperactivity whereas SD (low) was associated with inattention.

In conclusion, although concordant results emerged when comparing controls to ADHD subjects, this is not the case when looking at the different subtypes and severity of ADHD. In this perspective, we explored the association between ADHD subtypes and temperament and characters in adult life as well as that between these personality patterns and severity of the disease in a large community-based sample of adult ADHD cases compared to healthy controls. Based on the above-mentioned literature, we were expecting to find high NS, HA and ST scores as well as low SD and $C$ in subjects suffering from ADHD. We also hypothesized that deviation in NS and in HA would be related to hyperactive/impulsive and inattentive symptoms respectively. Finally, we were expecting to find an association between low SD and $\mathrm{C}$ scores with severity of ADHD.

\section{Methods}

\section{Participants}

One hundred and nineteen outpatients with adult ADHD were recruited in a specialized center for the diagnosis and care of adult ADHD patients at the University Hospitals of Geneva. ADHD diagnosis was made according to 
the DSM-IV criteria based on a clinical interview with trained psychiatrists (NP, PP, JZ and PB). Six or more symptoms of inattentive and/or hyperactive-impulsive symptoms were required and should be present before 7 years of age. The number of symptoms was used to determine the ADHD subtype as described by Prada et al. [28]. Briefly, a subject with 6 or more inattentive symptoms but less than 6 hyperactive-impulsive symptoms was classified as predominantly inattentive; a subject with 6 or more hyperactive-impulsive symptoms but less than 6 inattentive symptoms was classified as predominantly hyperactive-impulsive; and a subject with 6 or more hyperactive-impulsive and inattentive symptoms was classified as combined type.

Most of the adult ADHD patients $(\mathrm{N}=89)$ were addressed by either general practitioners or psychiatrists for an initial assessment and for a suspicion of ADHD and were thus free of any psychopharmacological treatment at the time of their recruitment; although some of them previously received antidepressants for previous major depressive disorder. The remaining 30 subjects were already diagnosed with ADHD and were, for most of them, taking psychostimulants when reaching our tertiary center.

The comparison group included 403 subjects recruited from the general population in the Blood Donor Center of the University Hospitals of Geneva and School of Dentistry at the University of Geneva.

Controls and ADHD patients with neurological conditions such as epilepsia were excluded from the study.

The study was approved by the ethics committee of Geneva University Hospitals. Informed written consent was obtained from all participants.

\section{Instruments}

A semi-structured interview assessing childhood and adulthood ADHD based on DSM-IV criteria: "Entretien diagnostique pour le TDAH chez l'adulte" (DIVA) [29] as well as the French version of the Diagnostic Interview for Genetic Studies (DIGS) [30] were administered to ADHD patients. The DIGS not only assesses Axis I comorbidities but also ADHD symptoms in childhood and their persistence into adulthood. In addition, all of the ADHD subjects completed the Wender Utah Rating Scale (WURS) [31], a self-report questionnaire assessing the severity of childhood ADHD, and the Adult ADHD Self-Report Scale (ASRS v1.1) [32], which assesses the severity of adult ADHD. They also fulfilled the Childhood Trauma Questionnaire (CTQ),which examines five types of trauma (sexual abuse, physical abuse, physical neglect, emotional abuse and emotional neglect) to assess childhood traumatic experience [33].

Finally, ADHD subjects completed the TCI. The TCI is a 240 -items (235 research items and 5 validation items for the assessment of response accuracy and carelessness) self-report questionnaire evaluating temperament and character dimensions. Each question should be answered by yes or no, assessing the four dimensions of temperament (novelty seeking, harm avoidance, reward dependence and persistence) and the three dimensions of character (self-directedness, cooperativeness and selftranscendence). Besides the 7 main scales, the TCI also provides a number of subscales ( 3 to 5 ) for each of the temperaments and characters (which will not be taken into account in the current paper). The Cronbach's alpha was good for each of the TCI subscales with values comprised between 0.89 and 0.93 .

All of the controls completed the TCI. Among them, 191 subjects were also screened for Axis I disorders for main diagnosis and comorbidities (142 negative), using the Mini International Neuropsychiatric Interview [34].

\section{Statistics}

One-way analysis of covariance (ANCOVA) with adjustment for age, included as a continuous was used to compare the Temperaments' and Characters' means between ADHD and controls.

Given the high frequency of psychiatric comorbidities in adults suffering from ADHD, and in order to test a "real life" hypothesis, two analyses were performed. The first concerned the whole sample: ADHD subjects without the exclusion of psychiatric comorbidities and all controls. In this analysis, controls corresponded to the notion of non-ADHD. The second analysis was made in ADHD subjects without psychiatric comorbidities and controls after exclusion of psychiatric disorders.

ANCOVA was also used to compare the Temperaments' and Characters' means between ADHD with the predominantly inattentive type and ADHD with the two other types (hyperactive/impulsive + combined). Given the small number of subjects suffering from the predominantly hyperactive/impulsive ADHD subtype $(\mathrm{n}=8)$, these subjects were pooled with subjects suffering from the combined subtype $(\mathrm{N}=65)$ for statistical purposes.

Finally, within ADHD subjects, partial correlation coefficients were computed between TCI temperaments and characters and ADHD symptoms, and severity with adjustment on age and gender. Partial correlation coefficients is calculated between one given variable (ex: NS) and a variable after removing the effects of all other variables entered into the model.

As characters are believed to be highly influenced by environmental factors, and thus vary during different stages of lifespan, we wondered whether childhood maltreatment would be associated with characters. Partial correlation coefficients were thus used to assess strength of correlation between different types of childhood 
maltreatments and TCI temperaments and characters within ADHD subjects.

When data was not normally distributed (namely HA, SD, ST, and P) Box-Cox transformation was used to take the appropriate remedial actions. This procedure helps to identify an appropriate exponent $($ Lambda $=1$ ) to use to transform data into a "normal shape." The power to which all data should be raised is determined by the Lambda value. To do so, the Box-Cox transformation seeks from Lambda $=-5$ to $\mathrm{Lamba}=+5$ until the best value is found. Then the appropriate action to transform the data is taken. STATA SE v.12.0 was used for the analyses and $p$ value of $\leq 0.05$ was considered as significant.

\section{Results}

Table 1 shows the TCI characters and temperaments of ADHD and control subjects. Compared to controls, ADHD diagnosis was associated with higher NS scores $\left(\mathrm{F}_{(1 / 521)}=28.3 ; \mathrm{p}<0.0001\right)$, higher HA scores $\left(\mathrm{F}_{(1 / 521)}=\right.$ 54.2; $\mathrm{p}<0.0001)$, higher ST scores $\left(\mathrm{F}_{(1 / 521)}=9.2 ; \mathrm{p}=\right.$ $0.003)$, lower SD scores $\left(\mathrm{F}_{(1 / 521)}=269.7 ; \mathrm{p}<0.0001\right)$ and lower $\mathrm{C}$ scores $\left(\mathrm{F}_{(1 / 521)}=26.4 ; \mathrm{p}<0.0001\right)$. Similar results were obtained (same magnitude of effect size) after exclusion of psychiatric comorbidities in ADHD subjects and exclusion of psychiatric disorders in controls (Table 1). Moreover, adding marital status as an environmental factors that may influence characters into the models did not change the results.

\section{Comparison of personality patterns in ADHD subtypes}

The ADHD predominantly inattentive type was related to lower ASRS v1.1 and WURS scores (Table 2). ADHD of the predominantly inattentive type was associated with lower NS scores and higher HA scores $\left(\mathrm{F}_{(1 / 118)}=\right.$ 9.3; $\mathrm{p}=0.003$ and $\mathrm{F}_{(1 / 118)}=5.2 ; \mathrm{p}=0.024$ ) (Table 2). Adding Axis 1 comorbidity (yes or no) as covariate led to similar results (data not shown).

\section{$\mathrm{TCl}$ correlation with ADHD symptoms and severity}

Table 3 shows the results of the partial correlation coefficients considering TCI temperaments and characters and ADHD symptoms and severity. Inattention symptoms positively correlated with $\mathrm{HA}$ and negatively with SD whereas hyperactive symptoms positively correlated with NS and ST. SD and C negatively correlated with the severity of ADHD in childhood. The severity of current ADHD was positively related to NS, RD, and ST and negatively correlated with SD.

\section{TCI correlation with childhood maltreatment}

None of the different types of childhood maltreatment did significantly correlate with TCI temperaments and characters (Table 4).

\section{Discussion}

In agreement with our hypotheses, our data revealed striking differences in both temperament and character dimensions between adult ADHD and healthy controls. We indeed found that high NS, HA and ST scores as well as low SD and $C$ scores were associated with ADHD diagnosis. We also found that high HA scores helped to distinguish the predominantly inattentive type from the pooled combined-hyperactive/impulsive subjects and that NS positively correlated with hyperactive symptoms. Finally, as expected, low SD scores were associated with increased ADHD severity.

Table 1 Panel A: Comparison of demographic and clinical characteristics of ADHD and control subjects for the whole sample; Panel B: Comparison of demographic and clinical characteristics of ADHD without Axis I comorbidities and control subjects without psychiatric disorders. For dimensional variables, ANCOVA were done with adjustment on age. TCI-R (Temperament and Character Inventory)

\begin{tabular}{|c|c|c|c|c|c|c|c|c|c|c|c|c|c|}
\hline & & \multicolumn{6}{|c|}{ A. Whole Sample } & \multicolumn{6}{|c|}{ B. Subjects without Axis I comorbidity(ADHD)/disorder(Controls) } \\
\hline & & \multicolumn{2}{|c|}{$\operatorname{ADHD}(n=119)$} & \multicolumn{2}{|c|}{ Controls $(n=403)$} & \multicolumn{2}{|c|}{ ADHD vs. Controls } & \multicolumn{2}{|c|}{$\operatorname{ADHD}(n=49)$} & \multicolumn{2}{|c|}{ Controls $(n=142)$} & \multicolumn{2}{|c|}{ ADHD vs. Controls } \\
\hline & & $N$ & $\%$ & $N$ & $\%$ & $\mathrm{OR}$ & $p$ & $N$ & $\%$ & $N$ & $\%$ & $\mathrm{OR}$ & $p$ \\
\hline \multirow{2}{*}{\multicolumn{2}{|c|}{ Female }} & 37 & 31.1 & 148 & 36.7 & 1.29 & 0.26 & 15 & 30.6 & 38 & 26.8 & 0.83 & 0.6 \\
\hline & & Mean & SD & Mean & SD & $F(d f)$ & $p$ & Mean & SD & Mean & SD & $F(d f)$ & $p$ \\
\hline \multicolumn{2}{|l|}{ Age } & 37.3 & 11 & 45.5 & 12.7 & $40.6(1 / 521)$ & $<0.0001$ & 35.6 & 12.8 & 45.5 & 13.2 & $28.8(1 / 260)$ & $<0.0001$ \\
\hline \multirow[t]{7}{*}{$\mathrm{TCl}-\mathrm{R}$} & NS & 23 & 5.9 & 18.8 & 5.6 & $28.3(1 / 521)$ & $<0.0001$ & 23.1 & 5.4 & 18.9 & 5.1 & $19.4(1 / 260)$ & $<0.0001$ \\
\hline & $\mathrm{HA}$ & 18.3 & 8.1 & 12.7 & 6.2 & $54.2(1 / 521)$ & $<0.0001$ & 16.5 & 8.5 & 11.1 & 5.5 & $50.4(1 / 260)$ & $<0.0001$ \\
\hline & $\mathrm{RD}$ & 14.2 & 4 & 14.6 & 3.8 & $2.8(1 / 521)$ & 0.096 & 13.7 & 3.5 & 14.3 & 4 & $0.6(1 / 260)$ & 0.458 \\
\hline & $P$ & 4.8 & 2 & 4.8 & 2 & $0.03(1 / 521)$ & 0.863 & 4.8 & 2.2 & 4.7 & 2 & $0.02(1 / 260)$ & 0.892 \\
\hline & ST & 15.3 & 6.8 & 13.5 & 6.3 & $9.2(1 / 521)$ & 0.003 & 15.1 & 7.2 & 13 & 6.3 & $8.4(1 / 260)$ & 0.004 \\
\hline & SD & 21.9 & 8.5 & 34.5 & 5.8 & $269.7(1 / 521)$ & $<0.0001$ & 23.4 & 8.7 & 35.3 & 5.4 & $161.4(1 / 260)$ & $<0.0001$ \\
\hline & C & 30.5 & 7.4 & 33.5 & 5 & $26.4(1 / 521)$ & $<0.0001$ & 30.1 & 6.1 & 33.7 & 4.5 & $14(1 / 260)$ & 0.0002 \\
\hline
\end{tabular}


Table 2 Clinical and demographic characteristics of ADHD subtypes

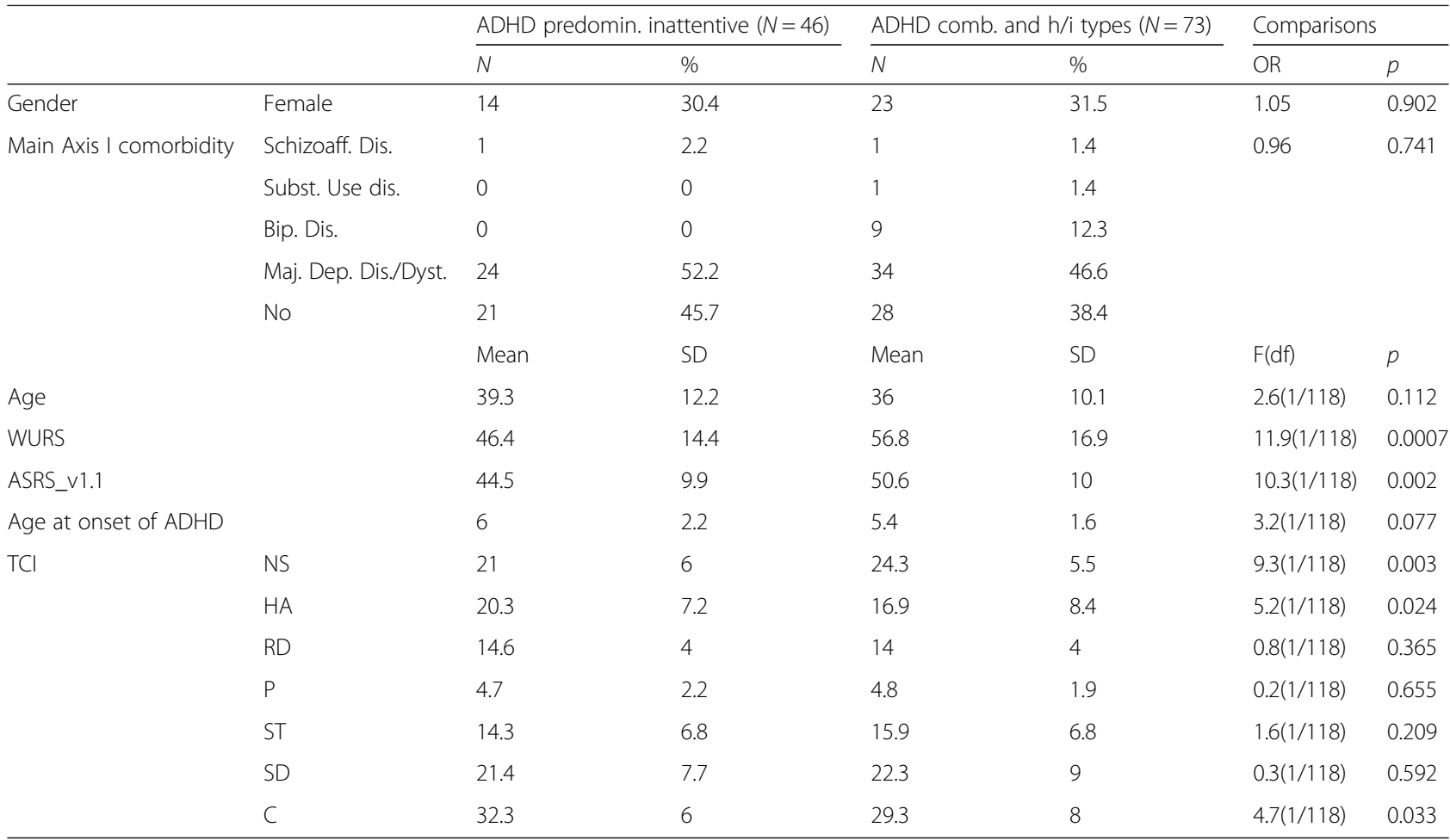

Abbreviations: NS Novelty Seeking, HA Harm Avoidance, RD Reward Dependence, P Persistence, ST Self-Transcendence, SD Self-Directedness, C Cooperativeness, TCI Temperament and Character Inventory, WURS Wender Utah Rating Scale, ASRS v1.1 Adult ADHD Self-Report Scale

Confirming previous investigations in this field [10, 11, 35-37], low SD and C scores were associated with ADHD diagnosis in the present series. SD reflects the individual's ability for autonomy, his/her reliability and maturity, how responsible, purposeful and resourceful he/she is and thus how this individual is able to achieve his/her own goal and to be autonomous [38]. Low scores on SD have been shown to predict personality disorders mainly from the cluster $\mathrm{B}$ and in particular borderline personality disorder $[16,20]$. In line with this idea, some authors have suggested that ADHD may represent a core vulnerability factor for borderline personality disorder in adolescence [39-42]. C indicates how well an individual is able to get along with others and closely reflects interpersonal skills such as empathy, compassions or the ability to be helpful. In addition to the lower SD, low $\mathrm{C}$ may thus relate to a deficit in maturity of personality and social skills and thus low psychological functioning and poor mental health outcomes $[10,18,37,43-45]$. Interestingly, SD negatively correlated

Table 3 A Partial correlation coefficients* between TCl temperaments and characters and number of ADHD symptoms. B. Partial correlation coefficients* between TCl temperaments and characters and continuous scales measuring severity of ADHD. Results with $\mathrm{p}<=0.05$ are bolded and italicized

\begin{tabular}{|c|c|c|c|c|c|c|c|}
\hline & NS & $\mathrm{HA}$ & $\mathrm{RD}$ & $P$ & ST & SD & $C$ \\
\hline & Coeff; $p$ & Coeff; $p$ & Coeff; $p$ & Coeff; $p$ & Coeff; $p$ & Coeff; $p$ & Coeff; $p$ \\
\hline \multicolumn{8}{|l|}{ A. } \\
\hline Inattention symptoms & $0.05 ; 0.55$ & $0.27 ; 0.003$ & $0.14 ; 0.12$ & $-0.14 ; 0.13$ & $0.10 ; 0.28$ & $-0.25 ; 0.007$ & $-0.13 ; 0.15$ \\
\hline Hyperactive symptoms & $0.21 ; 0.02$ & $-0.17 ; 0.06$ & $-0.01 ; 0.88$ & $-0.08 ; 0.39$ & $0.18 ; 0.05$ & $0.06 ; 0.48$ & $-0.08 ; 0.41$ \\
\hline Impulsive symptoms & $-0.07 ; 0.44$ & $-0.04 ; 0.65$ & $-0.04 ; 0.67$ & $0.16 ; 0.06$ & $-012 ; 0.20$ & $0.11 ; 0.24$ & $0.01 ; 0.97$ \\
\hline \multicolumn{8}{|l|}{ B. } \\
\hline WURS score & $-0.02 ; 0.86$ & $0.09 ; 0.35$ & $-0.11 ; 0.25$ & $-0.04 ; 0.63$ & $0.07 ; 0.45$ & $-0.31 ; 0.0008$ & $-0.27 ; 0.003$ \\
\hline ASRS-v1.1 score & $0.23 ; 0.01$ & $0.11 ; 0.25$ & $0.21 ; 0.02$ & $-0.02 ; 0.82$ & $0.19 ; 0.03$ & $-0.20 ; 0.03$ & $0.001 ; 0.90$ \\
\hline
\end{tabular}

Abbreviations: NS Novelty Seeking, HA Harm Avoidance, RD Reward Dependence, $P$ Persistence, ST Self-Transcendence, SD Self-Directedness, C Cooperativeness *Partial correlation coefficients is calculated between one given variable (ex: NS) and a variable after removing the effects of all other variables entered into the model 
Table 4 Partial correlation coefficients* between TCl temperaments and characters and different types of childhood maltreatments after adjustment on age and gender

\begin{tabular}{llllllll}
\hline & NS & HA & RD & $P$ & ST & $\begin{array}{l}\text { SD } \\
\text { Coeff; } p\end{array}$ & $\begin{array}{l}\text { C } \\
\text { Coeff; } p\end{array}$ \\
& Coeff; $p$ & Coeff; $p$ & Coeff; $p$ & Coeff; $p$ & Coeff; $p$ & $-0.04 ; 0.60$ & $-0.10 ; 0.26$ \\
\hline Emotional neglect & $-0.15 ; 0.12$ & $0.14 ; 0.12$ & $-0.13 ; 0.16$ & $0.11 ; 0.22$ & $-0.07 ; 0.41$ & $-0.0 .05 ; 0.59$ & $-0.05 ; 0.56$ \\
Emotional abuse & $-0.01 ; 0.97$ & $-0.07 ; 0.41$ & $-0.01 ; 0.87$ & $-0.04 ; 0.67$ & $-0.02 ; 0.83$ & -0.07 \\
Physical neglect & $0.10 ; 0.29$ & $0.06 ; 0.51$ & $0.01 ; 0.96$ & $-0.06 ; 0.46$ & $0.13 ; 0.15$ & $-0.04 ; 0.61$ & $0.01 ; 0.98$ \\
Physical abuse & $-0.03 ; 0.72$ & $0.01 ; 0.95$ & $-0.16 ; 0.09$ & $0.12 ; 0.19$ & $0.11 ; 0.23$ & $-0.01 ; 0.91$ & $-0.02 ; 0.79$ \\
Sexual abuse & $0.02 ; 0.81$ & $0.02 ; 0.48$ & $0.15 ; 0.11$ & $-0.15 ; 0.11$ & $0.13 ; 0.14$ & $0.08 ; 0.63$ & $0.12 ; 0.18$ \\
\hline
\end{tabular}

Abbreviations: NS Novelty Seeking, HA Harm Avoidance, RD Reward Dependence, $P$ Persistence, ST Self-Transcendence, SD Self-Directedness, C Cooperativeness

with the severity of ADHD in adulthood and childhood and, concordant with previous findings, a negative correlation between $\mathrm{C}$ and severity of ADHD in childhood was also found $[11,15,35]$. In this perspective, Martel et al. found that, in children, low $\mathrm{C}$ partly mediated the effect of genetic factors on ADHD symptoms with a main impact on the inattentive subtype [45]. Taken together, these results indicate that character immaturity is associated with ADHD severity possibly leading to the paucity of interpersonal relationships observed in severe forms of this disorder $[11,13,37]$. Whether this association reflects early neurodevelopmental deficits is still unclear in the absence of longitudinal data.

Not surprisingly, high NS scores were also found in ADHD subjects compared to controls [12, 14-16, 18, 37, 46]. However, and in contrast to previous studies, this association was modest in our cases [14, 15]. NS mainly reflects individual variability in initiating a behavior in response to stimuli and comprises impulsiveness, the search for novel and unknown experiences, but also spontaneity and extraversion in social situations. According to Cloninger [47], people with high NS are impulsive, curious, disordered, extravagant, easily bored and hot-headed, which is, by definition, a trait expected to be higher in ADHD subjects than in controls. As previously reported, NS positively correlated with ADHD severity and hyperactive symptoms $[11,13,16,19,23,26]$. High NS scores are indeed rather related to impulsivity/hyperactivity rather than inattentive problems $[19,26]$. It is thus not surprising to find that, in our study, NS enabled to distinguish the predominantly inattentive type from the pooled combined-hyperactive/impulsive subjects with the latter displaying the highest scores.

Several previous reports found higher HA in ADHD compared to controls [4, 14-17, 35, 37]. Our results were consistent with these findings showing that higher HA was associated with ADHD. This association persist after controlling for psychiatric comorbidities such as anxiety, depression and substance use disorders [12, 18]. Our findings suggest that ADHD subjects are, independently of other psychiatric comorbidities, easily worried, fearful, shy, socially reserved, and easily tired $[1,48]$. In addition, higher HA scores enabled to distinguish the predominantly inattentive type from the pooled combinedhyperactive/impulsive subjects $[13,23]$. In the same line, recent research showed a robust association between HA and inattentive symptoms, primarily due to overlapping genetic influences [26]. As suggested by Merwood et al., this association may be suggestive of an increased risk for internalizing symptoms such as anxiety disorders in ADHD subjects with higher number of inattentive symptoms $[26,49]$.

Last but not least, higher ST scores were found in ADHD subjects compared to controls. Moreover, ST positively correlated with ADHD severity in adulthood. In agreement with previous reports, these results support the idea that ADHD subjects tend to be spiritual, satisfied, modest and self-forgetful $[17,18]$. Intriguingly, high ST scores have been found in several psychiatric entities suggesting that ST may be a vulnerability trait for neuropsychiatric disorders. In the Cloninger's theory, high ST combined with low scores on the two other characters is associated with schizotypal and paranoid symptoms and may provide a good proxy for the existence of a personality disorder [50]. It has to be kept in mind that spirituality and religion may help patients to cope with their illness. If this has been more deeply investigated for disorders such as schizophrenia, this has not been investigated in relation to ADHD and further researches are needed in this field [51].

Although previous studies found low RD in ADHD subjects [17], suggesting that they are socially insensible and indifferent, our results did not corroborate this viewpoint. ADHD subjects scored roughly the same as controls on RD indicating that they did not differ from the general population in terms of attachment and dependence.

This study has several limitations, starting with the relative small sample size of ADHD subtypes. Secondarily, this study relies on self-report questionnaires assessing personality traits that are hypothesized to reflect the individual's characters and temperaments throughout the course of his/her life. This hampers the possibility of determining the temporal relationship between personality changes and ADHD occurrence. Thirdly, only a fraction of our controls were assessed for psychiatric disorders and 
comorbidities, and thus some of them may still suffer from ADHD or other psychiatric disorders that have not been identified. Our separate analysis showed that the exclusion of psychiatric comorbidities in both groups did not alter the observed associations. Moreover, more that $70 \%$ of the screened controls did not have any psychiatric disorder further decreasing the probability of erroneous assumptions due to comorbidities. In addition, we did not assess several other clinical and demographic variables potentially affecting TCI dimensions and more specifically characters that are believed to be highly associated with environmental influences. We were thus not able to adjust for such variables and this caveat should be kept in mind when interpreting our findings. Finally, we did not control for the fact that some ADHD patients took psychostimulants in childhood and that this may have affected the development of their personality.

\section{Conclusions}

The present observations suggest that ADHD is associated with specific personality traits which may reflect poor mental health outcomes. Our results are concordant with previous contributions using the Five Factor Model (FFM) of personality showing that ADHD subjects whatever is their age display deviation in several personality dimensions [27]. In a recent and elegant meta-analytic review, Gomez and Corr have shown, using the integrated FFM proposed by Markon et al. [52] which includes the personality dimensions of several questionnaires including the FFM and the TCI, that ADHD subjects compared to controls have low levels of conscientious inhibition which may be interpreted as high level of NS, low levels of agreeable inhibition (roughly corresponding to $\mathrm{C}$ ), and high levels of negative emotionality which includes the TCI temperament $\mathrm{HA}$ and is inversely correlated to SD. As mentioned above, determining whether characters and temperaments are risk factors for the development of ADHD or the opposite deserves further investigation. However temperaments have been constructed as biological dimensions emerging early in life and thus possibly before the onset of ADHD. This may not be the case for characters that often reflect the influence of ADHD on personality development. Therefore, assuming that temperaments precede the onset of ADHD, targeting NS in childhood may provide an interesting predictor for identifying at risk ADHD subjects.

Our results also reveal that HA and NS scores may differentiate inattentive from hyperactive/impulsive ADHD subtypes [44, 53, 54]. Following Merwood et al., we may propose that the individual's profile of temperaments may help identify ADHD subtypes possibly better than relying only on a clinical evaluation [26].

In conclusion, our study offers a compelling picture of personality deviations in ADHD and how they relate to symptom dimensions. Longitudinal studies are clearly needed in this field in order to better understand the complex interplay between personality and ADHD symptoms.

\section{Abbreviations \\ ADHD, Attention-deficit hyperactivity disorder; ANCOVA, One-way analysis of covariance; ASRS, Adult ADHD Self-Report Scale; C, cooperativeness; CTQ \\ Childhood Trauma Questionnaire; DIGS, Diagnostic Interview for Genetic Studies; DIVA, Entretien diagnostique pour le TDAH chez l'adulte; FFM, Five Factor Model; HA, harm avoidance; NS, High novelty seeking; P, persistence; $\mathrm{RD}$, reward dependence; SD, self-directedness; ST, self-transcendence; TCl, Temperament and Character Inventory; WURS, Wender Utah Rating Scale.}

\section{Acknowledgements}

We would like to thank Gérald Bouillault, Venus Kaby, Jean-Jacques Kunckler, Karen Dieben, Caroline Waeber, and Sophie Blin.

\section{Funding}

None.

Availability of data and materials

Shared upon request.

Authors' contributions

NP and PP drafted the manuscript. NP and PB conceptualized and designed the study. NP and FH performed the statistical analyses and interpreted the data. PG and JMA interpreted the data and helped in the drafting the manuscript. PG, JMA, NG, SA, JZ, RH and RN participated in the design of the study and in the acquisition of data. All authors read and approved the final manuscript.

\section{Competing interests}

NP and JMA received honoraria for talks from several pharmaceutical agencies including Lundbeck, Elli-Lilly and AstraZeneka.

\section{Consent for publication}

Not applicable.

\section{Ethics approval and consent to participate}

The study was approved by the ethics committee of Geneva University Hospitals. Informed written consent was obtained from all participants.

\section{Author details}

'Department of Mental Health and Psychiatry, University Hospitals of Geneva, Geneva, Switzerland. 'Department of Psychiatry, University of Geneva, Geneva, Switzerland. ${ }^{3}$ Section of Dental Medicine, Faculty of Medicine, University of Geneva, Geneva, Switzerland. ${ }^{4}$ Division of Geriatrics, Department of Internal Medicine, Rehabilitation and Geriatrics, Geneva University Hospitals and University of Geneva, Geneva, Switzerland.

Received: 22 April 2015 Accepted: 15 April 2016

Published online: 14 June 2016

\section{References}

1. Kessler RC, Adler L, Barkley R, Biederman J, Conners CK, Demler O, et al. The prevalence and correlates of adult ADHD in the United States: results from the National Comorbidity Survey Replication. Am J Psychiatry. 2006;163(4):716-23.

2. Miller CJ, Miller SR, Newcorn JH, Halperin JM. Personality characteristics associated with persistent ADHD in late adolescence. J Abnorm Child Psychol. 2008;36(2):165-73

3. May B, Bos J. Personality characteristics of ADHD adults assessed with the Millon Clinical Multiaxial Inventory-ll: evidence of four distinct subtypes. J Pers Assess. 2000;75(2):237-48.

4. Jacob CP, Romanos J, Dempfle A, Heine M, Windemuth-Kieselbach C, Kruse A, et al. Co-morbidity of adult attention-deficit/hyperactivity disorder with focus on personality traits and related disorders in a tertiary referral center. Eur Arch Psychiatry Clin Neurosci. 2007;257(6):309-17.

5. Cloninger CR, Svrakic DM, Przybeck TR. A psychobiological model of temperament and character. Arch Gen Psychiatry. 1993;50(12):975-90. 
6. Ando J, Suzuki A, Yamagata S, Kijima N, Maekawa H, Ono Y, et al. Genetic and environmental structure of Cloninger's temperament and character dimensions. J Pers Disord. 2004;18(4):379-93.

7. Stallings MC, Hewitt JK, Cloninger CR, Heath AC, Eaves LJ. Genetic and environmental structure of the Tridimensional Personality Questionnaire: three or four temperament dimensions? J Pers Soc Psychol. 1996;70(1):127-40.

8. Cloninger CR, editor. The genetics and psychobiology of the seven-factor model of personality. Washington, DC: American Psychiatric Press; 1998.

9. Instanes JT, Haavik J, Halmoy A: Personality Traits and Comorbidity in Adults With ADHD. J Atten Disord. 2013. doi:10.1177/1087054713511986.

10. Purper-Ouakil D, Cortese S, Wohl M, Aubron V, Orejarena S, Michel G, et al. Temperament and character dimensions associated with clinical characteristics and treatment outcome in attention-deficit/hyperactivity disorder boys. Compr Psychiatry. 2010;51(3):286-92.

11. Cho SC, Hwang JW, Lyoo IK, Yoo HJ, Kin BN, Kim JW. Patterns of temperament and character in a clinical sample of Korean children with attention-deficit hyperactivity disorder. Psychiatry Clin Neurosci. 2008;62(2):160-6.

12. Tillman R, Geller B, Craney JL, Bolhofner K, Williams M, Zimerman B, et al. Temperament and character factors in a prepubertal and early adolescent bipolar disorder phenotype compared to attention deficit hyperactive and normal controls. J Child Adolesc Psychopharmacol. 2003;13(4):531-43.

13. Salgado CA, Bau CH, Grevet EH, Fischer AG, Victor MM, Kalil KL, et al. Inattention and hyperactivity dimensions of ADHD are associated with different personality profiles. Psychopathology. 2009;42(2):108-12.

14. Downey KK, Stelson FW, Pomerleau OF, Giordani B. Adult attention deficit hyperactivity disorder: psychological test profiles in a clinical population. J Nerv Ment Dis. 1997;185(1):32-8.

15. Lynn DE, Lubke G, Yang M, McCracken JT, Mcgough JJ, Ishii J, et al. Temperament and character profiles and the dopamine D4 receptor gene in ADHD. Am J Psychiatry. 2005;162(5):906-13.

16. Anckarsater H, Stahlberg O, Larson T, Hakansson C, Jutblad SB, Niklasson L, et al. The impact of ADHD and autism spectrum disorders on temperament, character, and personality development. Am J Psychiatry. 2006;163(7):1239-44.

17. Faraone SV, Kunwar A, Adamson J, Biederman J. Personality traits among ADHD adults: implications of late-onset and subthreshold diagnoses. Psychol Med. 2009:39(4):685-93.

18. Sizoo B, van den Brink W, Gorissen van Eenige M, van der Gaag Brink RJ Personality characteristics of adults with autism spectrum disorders or attention deficit hyperactivity disorder with and without substance use disorders. J Nerv Ment Dis. 2009;197(6):450-4.

19. Rettew DC, Copeland W, Stanger C, Hudziak JJ. Associations between temperament and DSM-IV externalizing disorders in children and adolescents. J Dev Behav Pediatr. 2004;25(6):383-91.

20. van Dijk FE, Lappenschaar M, Kan CC, Verkes RJ, Buitelaar JK. Symptomatic overlap between attention-deficit/hyperactivity disorder and borderline personality disorder in women: the role of temperament and character traits. Compr Psychiatry. 2012:53(1):39-47.

21. Kim HW, Cho SC, Kim BN, Kim JW, Shin MS, Yeo JY. Does oppositional defiant disorder have temperament and psychopathological profiles independent of attention deficit/hyperactivity disorder? Compr Psychiatry. 2010:51(4):412-8.

22. Cho SC, Kim BN, Kim JW, Rohde LA, Hwang JW, Chungh DS, et al. Full syndrome and subthreshold attention-deficit/hyperactivity disorder in a Korean community sample: comorbidity and temperament findings. Eur Child Adolesc Psychiatry. 2009:18(7):447-57.

23. Gomez R, Woodworth R, Waugh M, Corr PJ. Attention-deficit/hyperactivity disorder symptoms in anadult sample: associations with Cloninger's temperament and character dimensions. Pers Individ Differ. 2012;52:290-4

24. Wood AC, Rijsdijk F, Asherson P, Kuntsi J. Inferring Causation from Cross-Sectional Data: Examination of the Causal Relationship between Hyperactivity-Impulsivity and Novelty Seeking. Front Genet. 2011;2:6.

25. Young SE, Friedman NP, Miyake A, Willcutt EG, Corley RP, Haberstick BC, et al. Behavioral disinhibition: liability for externalizing spectrum disorders and its genetic and environmental relation to response inhibition across adolescence. J Abnorm Psychol. 2009;118(1):117-30.

26. Merwood A, Asherson P, Larsson H. Genetic associations between the ADHD symptom dimensions and Cloninger's temperament dimensions in adult twins. Eur Neuropsychopharmacol. 2013;23(6):416-25.

27. Stoffers JM, Vollm BA, Rucker G, Timmer A, Huband N, Lieb K. Psychological therapies for people with borderline personality disorder. Cochrane Database Syst Rev. 2012;8, CD005652
28. Prada P, Hasler R, Baud P, Bednarz G, Ardu S, Krejci I, et al. Distinguishing borderline personality disorder from adult attention deficit/hyperactivity disorder: a clinical and dimensional perspective. Psychiatry Res. 2014;217(1-2):107-14

29. DIVA Foundation: Entretien diagnostique pour le TDAH chez l'adulte (DIVA), [www.divacenter.eu/Content/Downloads/DIVA_2_FRANCAIS.pdf], 2012. Accessed 10 Jun 2016

30. Preisig M, Fenton BT, Matthey ML, Berney A, Ferrero F. Diagnostic interview for genetic studies (DIGS): inter-rater and test-retest reliability of the French version. Eur Arch Psychiatry Clin Neurosci. 1999;249(4):174-9.

31. Ward MF, Wender PH, Reimherr FW. The Wender Utah Rating Scale: an aid in the retrospective diagnosis of childhood attention deficit hyperactivity disorder. Am J Psychiatry. 1993;150(6):885-90.

32. Romo L, Legauffre C, Mille S, Cheze N, Fougeres AL, Marquez S, et al. [Psychometric properties of the French version of the Wender Utah Rating Scale and Brown's Attention Deficit Disorders Scale for adults]. Encéphale. 2010;36(5):380-9.

33. Bernstein DP, Fink L. Childhood Trauma Questionnaire. ARetrospective Self-Report. San Antonio, USA: The Psychological Corporation; 1998.

34. Sheehan DV, Lecrubier $Y$, Sheehan KH, Amorim P, Janavs J, Weiller $E$, Hergueta T, Baker R, Dunbar GC. The Mini-International Neuropsychiatric Interview (M.I.N.I.): the development and validation of a structured diagnostic psychiatric interview for DSM-IV and ICD-10. J Clin Psychiatry. 1998;59 Suppl 20:22-33

35. Muller DJ, Chiesa A, Mandelli L, De Luca V, De Ronchi D, Jain U, et al. Correlation of a set of gene variants, life events and personality features on adult ADHD severity. J Psychiatr Res. 2010;44(9):598-604.

36. Garcia D, Anckarsater H, Lundstrom S. Self-directedness and cooperativeness, psychosocial dysfunction and suffering in ESSENCE. ScientificWorldJournal. 2013:2013:416981.

37. Kerekes N, Brandstrom S, Lundstrom S, Rastam M, Nilsson T, Anckarsater H. $A D H D$, autism spectrum disorder, temperament, and character: phenotypical associations and etiology in a Swedish childhood twin study. Compr Psychiatry. 2013:54(8):1140-7.

38. Cloninger CR. Temperament and personality. Curr Opin Neurobiol. 1994:4(2):266-73.

39. Davids E, Gastpar M. Attention deficit hyperactivity disorder and borderline personality disorder. Prog Neuropsychopharmacol Biol Psychiatry. 2005;29(6):865-77.

40. Philipsen A. Differential diagnosis and comorbidity of attention-deficit/ hyperactivity disorder (ADHD) and borderline personality disorder (BPD) in adults. Eur Arch Psychiatry Clin Neurosci. 2006;256 Suppl 1:i42-6.

41. Matthies S, van Elst LT, Feige B, Fischer D, Scheel C, Krogmann E, et al. Severity of childhood attention-deficit hyperactivity disorder-a risk factor for personality disorders in adult life? J Pers Disord. 2011;25(1):101-14.

42. Speranza M, Revah-Levy A, Cortese S, Falissard B, Pham-Scottez A, Corcos M ADHD in adolescents with borderline personality disorder. BMC Psychiatry. 2011;11:158

43. Nigg JT, Goldsmith HH, Sachek J. Temperament and attention deficit hyperactivity disorder: the development of a multiple pathway model. J Clin Child Adolesc Psychol. 2004:33(1):42-53.

44. Martel MM, Nigg JT, von Eye A. How do trait dimensions map onto ADHD symptom domains? J Abnorm Child Psychol. 2009;37(3):337-48.

45. Martel MM, Nikolas M, Jernigan K, Friderici K, Nigg JT. Personality mediation of genetic effects on Attention-Deficit/Hyperactivity Disorder. J Abnorm Child Psychol. 2010;38(5):633-43.

46. Guimaraes-Da-Silva PO, Silva KL, Grevet EH, Salgado CA, Karam RG, Victor MM, et al. Does age of onset of impairment impact on neuropsychological and personality features of adult ADHD? J Psychiatr Res. 2012:46(10):1307-11.

47. Cloninger CR. Neurogenetic adaptive mechanisms in alcoholism. Science. 1987:236(4800):410-6.

48. Biederman J, Monuteaux MC, Mick E, Spencer T, Wilens TE, Silva JM, et al. Young adult outcome of attention deficit hyperactivity disorder: a controlled 10-year follow-up study. Psychol Med. 2006;36(2):167-79.

49. Acosta MT, Castellanos FX, Bolton KL, Balog JZ, Eagen P, Nee L, et al. Latent class subtyping of attention-deficit/hyperactivity disorder and comorbid conditions. J Am Acad Child Adolesc Psychiatry, 2008:47(7):797-807.

50. Svrakic DM, Draganic S, Hill K, Bayon C, Przybeck TR, Cloninger CR. Temperament, character, and personality disorders: etiologic, diagnostic, treatment issues. Acta Psychiatr Scand. 2002;106(3):189-95. 
51. Mohr S, Borras L, Nolan J, Gillieron C, Brandt PY, Eytan A, et al. Spirituality and religion in outpatients with schizophrenia: a multi-site comparative study of Switzerland, Canada, and the United States. Int J Psychiatry Med. 2012;44(1):29-52.

52. Bateman AW, Fonagy P. Mentalization-based treatment of BPD. J Pers Disord. 2004;18(1):36-51.

53. Mcloughlin G, Ronald A, Kuntsi J, Asherson P, Plomin R. Genetic support for the dual nature of attention deficit hyperactivity disorder: substantial genetic overlap between the inattentive and hyperactive-impulsive components, J Abnorm Child Psychol. 2007;35(6):999-1008.

54. Nigg JT, John OP, Blaskey LG, Huang-Pollock CL, Willcutt EG, Hinshaw SP, et al. Big five dimensions and ADHD symptoms: links between personality traits and clinical symptoms. J Pers Soc Psychol. 2002;83(2):451-69.

Submit your next manuscript to BioMed Central and we will help you at every step:

- We accept pre-submission inquiries

- Our selector tool helps you to find the most relevant journal

- We provide round the clock customer support

- Convenient online submission

- Thorough peer review

- Inclusion in PubMed and all major indexing services

- Maximum visibility for your research

Submit your manuscript at www.biomedcentral.com/submit
Biomed Central 